\title{
FLOW ANALYSIS OF HYDRAULIC POPPET CONTROL VALVE BY MEANS OF COMPUTIONAL FLUID DYNAMICS
}

\author{
Jacek Lazowski \\ SKA-Polska, Sp. z o.o. \\ Jerozolimskie Av. 125/127 p. 406 02-017 Warsaw, Poland \\ tel./fax: +48226321775 \\ e-mail:j.lazowski@ska-polska.pl \\ Janusz Krasucki \\ CIM-mes Projekt, Sp. z o.o. \\ Jerozolimskie Av. 125/127 p. 503 02-017 Warsaw, Poland \\ tel.: +48226312244 \\ e-mail: j.krasucki@cim-mes.com.pl
}

\begin{abstract}
The poppet control valve is one of the most widespread hydraulic components. The paper purpose is the evaluation of flow characteristics of poppet valve by means of a complete numerical analysis. The aim of this analysis is to evaluate the valve fluid dynamic performance, exploiting computational fluid dynamics (CFD) techniques, in order to give the reliable indications needed to define the valve design criteria and avoid expensive experimental tests. The numerical analysis was performed by using the commercial code of CFD, and the numerical results show the complete flow field inside the valve. Axisymmetric as well as three-dimensional valve model was considered and the simulation results also are verified with experimental results. The results have confirmed the good accuracy of the simplified CFD analysis based on 2D axisymmetric valve model being more efficient than full 3D approach and show this method as very useful to forecast flow characteristics of poppet control valves.

Hydraulic control poppet valve, distribution of the velocity in layers of the fluid, computational grid in the modelled geometry, pressure distribution for axisymmetric and three-dimensional mode for different flow rate, fluid velocity distribution in valve metering section for axisymmetric and three-dimensional model for different flow rates, the example of streamlines, the comparison of flow characteristics are contained in the publication.
\end{abstract}

Keywords: poppet control valve, computational fluid dynamics, flow characteristics

\section{Introduction}

The performance of a hydraulic control system is strongly influenced by the dynamic characteristics of its control valves. The function of a control valve is to change the flow paths in hydraulic circuit, in particularly to open or to close the flow path. The control valve is one of the most expensive and sensitive parts of a hydraulic circuit and therefore must be designed very carefully.

In a classical approach, a many experimental measurements on the specific valve are needed. Alternatively, a numerical analysis can be performed by means of computational fluid dynamics (CFD) codes. Computational fluid dynamics (CFD) is becoming a well established practice also in valve analysis and design [3], since it can give a clear insight into its operation mode, which cannot be achieved by experimental tests and measurements. CFD also allows a reduction in the number of prototypes under test, as well as the time and costs of the design and of the experimental phase.

The difficulties introduced by the use of CFD techniques deal with the necessity to reduce the computational domain to the most important regions of the flow where the fluid dynamic phenomena are concentrated in order not to exceed the available computational resources. In fact, the traditional approach is axisymmetric modelling of the valve. This approximation allows the use of a more refined grid and a more accurate analysis of the flow field inside the valve. 
It requires assuming of some simplification in particular the geometry of set of circular inlet holes placed in circumference of valve housing should be replaced by one equivalent circumference channel. In this work, the investigations have been carried out on the example of control ON-OFF valve 2URES6 by polish manufacturer "PONAR" Wadowice.

Within the work, the comparison of 3D CFD analysis based on three-dimensional valve model and simplified approach using axisymmetric model was conducted. The simulation results were compared with experimental flow characteristic of the valve[2].

\section{The hydraulic control poppet valve}

Two way control poppet valves are pilot operated, low leakage solenoid actuated valves. Two way poppet valves control the flow of a two way function by blocking flow in one direction (similar to a check valve). They are generally selected due to their low leakage and ability to meet higher flow requirements. Poppet valves are often used on single operation actuators or in unloading functions. They are available in normally closed and normally open types. Normally closed poppet valves act as a check valve when de-energized, blocking flow from one direction and allowing restricted free flow in the reverse condition.

The scheme of analyzed valve type 2URES6 is shown in Fig. 1.

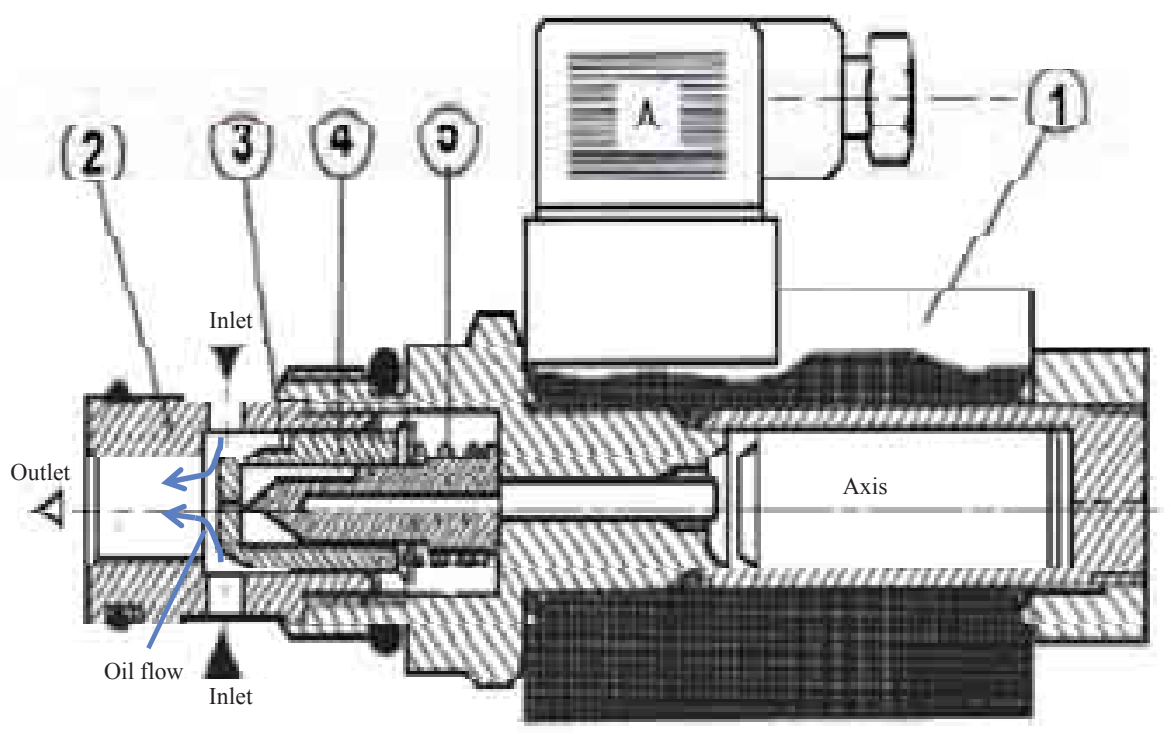

Fig. 1. The hydraulic control poppet valve 2URES6; 1-solenoid, 2-sleeve, 3 - poppet, 4 - pilot, 5 -spring

The valve pilot 4 is held on its seat by spring force 5, blocking pilot flow. This allows pressure at the inlet port to hold the poppet 4 on its seat, thus, preventing flow through the valve. If the nose of the inlet port is pressurized, the pressure will overcome the spring force 5, pushing the poppet 4 off its seat, allowing free flow through the valve. When the coil 1 is energized, the valve pilot 4 is pulled off its seat. This vents the pressure inside the poppet to inlet port, creating a pressure imbalance across the main poppet. This differential lifts the poppet 4 allowing flow from the side to nose. Since poppet valves are piloted operated, a minimum amount of pressure differential and flow between inlet and outlet ports must be present to overcome the spring and lift the poppet.

The flow through the valve orifice arises the pressure drop, which is considered as a difference between inlet and outlet pressure. The pressure drop results from fluid energy dissipation as a result of change flow velocity and internal friction.

That energy part can be dissipated in the process formation of vortices and internal friction and can be expressed mathematically as a coefficient of the hydraulic losses. The change of direction of fluid velocity causes of hydrodynamic force acting on the poppet 3 in direction of valve closing. 
The flow through the valve is turbulent and the relationship between pressure drop and flow rate is nonlinear and has nature of parabolic function $[1,6]$.

The one of the most important things, which should be considered by valve designers, is to minimize the pressure drops and to keep the hydrodynamic force in the lowest value, as it is possible.

\section{Physical model of the flow}

The choice of the physical model should reflect the most phenomena in the object that allowing to attain posed the purpose of the work. In this work, the main flow through the valve orifice between the main poppet and the valve seat was considered. I was assumed the flow is uniform and steady-state and working fluid is incompressible [4].

Properties of the working medium were modelled as for the Newtonian fluid in which the dynamic viscosity $\mu$ is independent of the velocity of the fluid. It assumes that the coefficient of viscosity is also independent from the temperature and the pressure.

A proper estimation of turbulent phenomena has great importance to determine the valve flow features. In particular, the flow inside a hydraulic valve is characterized by the coexistence of "free shear flows", due to the flow jet at the exit of the metering section, and "wall bounded flows", which are strongly influenced by the wall effects. The most suitable turbulence model for this kind of problem appears to be the RNG-k-e model coupled with the so called "two layer zonal model" [1, 4].

The most suitable turbulence model for this kind. In the paper the k- $\varepsilon$. model of turbulence was assumed because is suitable for the flow analysis of incompressible fluid with low velocity $[1,4]$. This turbulence model using the mean component method and based on the Reynolds rules gives a reliable estimation of the turbulent quantities upstream and downstream of the restricted sections and is able to estimate properly both the free jet and the wall bounded region.

\section{Mathematical model of the flow}

The homogeneous, incompressible and viscous flow of a working medium through the control valve is described by the Navier-Stokes equations (N-S) (1) for the steady-state flow. N-S equation together with the continuity equation (2) is the complete set of equations that determine the flow pressure and velocity fields.

Equation N-S can be written in the vector form $[1,3]$ :

$$
\vec{F}-\frac{1}{\rho} \operatorname{grad} p+v \Delta \vec{V}=0
$$

and the equation of continuity:

$$
\operatorname{div}(\rho \vec{V})=0
$$

where:

$\rho$ - fluid density $\left[\mathrm{kg} / \mathrm{m}^{3}\right]$

$\mathrm{p}$ - pressure $[\mathrm{Pa}]$,

$\vec{F}$ - vector of the massive force $[\mathrm{N}]$,

$v$ - coefficient of kinematical viscosity $\left[\mathrm{m}^{2} / \mathrm{s}\right]$,

$\vec{V}$ - velocity vector of control volume $[\mathrm{m} / \mathrm{s}]$.

Shear stress inside the fluid is expressed as:

$$
\tau=\mu \frac{d u}{d x}
$$

where: 
$\tau \quad$ - shearing stress in the liquid $[\mathrm{Pa}]$,

$\mu \quad$ - coefficient of dynamic viscosity [Pa.s],

$\mathrm{du} / \mathrm{dx}$ - shear speed $[\mathrm{m} / \mathrm{s}]$.

The velocity distribution across the fluid layers is illustrated by Fig. 2 .

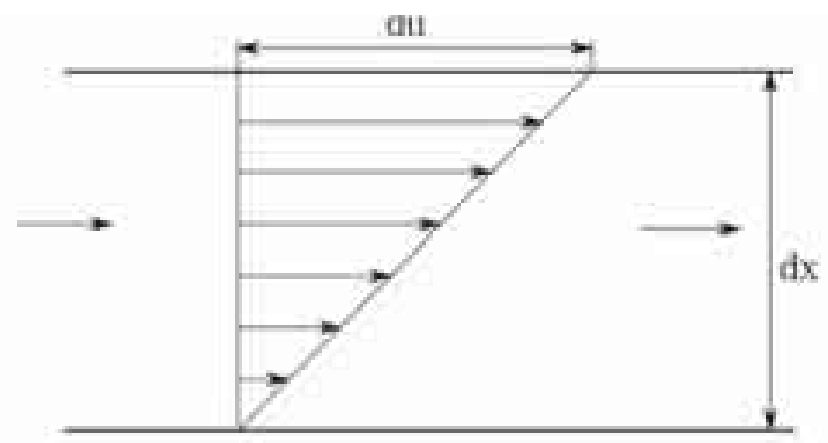

Fig. 2. Distribution of the velocity in layers of the fluid

Velocity profile across the fluid layers, shown in Fig. 2, causes the momentum differences between a fluid layers and in effect the shear stresses inside the fluid.

The components of Reynolds stress tensor is expressed by equation $[1,3]$ :

$$
\tau_{i j}^{*}=\mu_{T}\left(u_{u, j}+u_{j, i}\right)-\frac{2}{3} \rho k \delta_{i j}, \quad \mu_{T}=\rho c_{\mu} k^{2} / \varepsilon,
$$

where:

$k$ - denote kinetic energy [J],

$\varepsilon$ - denote dissipation of kinetic energy,

$c_{\mu}$ - constant.

The $k, \varepsilon$ parameters are related by following equations $[1,4]$ :

$$
\begin{aligned}
& \left(\rho k u_{i}\right)_{, i}=\left(\tau_{i j} u_{j}\right)_{, i}-\rho \varepsilon+\left(\mu_{k} k_{, i}\right)_{, i}, \\
& \left(\rho \varepsilon u_{i}\right)_{, i}=c_{\varepsilon 1}\left(\tau_{i j} u_{j}\right)_{, i}-\frac{c_{\varepsilon 2} \rho \varepsilon^{2}}{k}+\left(\mu_{\varepsilon} \varepsilon_{, i}\right)_{, i},
\end{aligned}
$$

while: $\mu_{k}=\mu+\mu_{T} / \sigma_{k}, \mu_{\varepsilon}=\mu+\mu_{T} / \sigma_{\varepsilon}$

and constants value:

$$
\mathrm{c}_{\mu}=0.09, \mathrm{c}_{\varepsilon 1}=1.45-1.55, \mathrm{c}_{\varepsilon 2}=1.92-2.00, \sigma_{\mathrm{k}}=1.00 ; \sigma_{\varepsilon}=1.30 .
$$

\section{Computational model}

The whole computational grid for two kinds of considered flow models is shown in Fig. 3. The boundary conditions are assumed as follows:

- INLET: on this surface, the constant pump flow rate value has been enforced and in consequence a constant inlet velocity of the medium,

- OUTLET: on this surface, the pressure value set by the pressure relief valve on the charge pump line has been imposed; for simple interpretation of the results the $p=0$ value may be accepted too,

- SYMMETRY: these are the symmetry faces and allow the use of the simplified geometry as previously described, then axisymmetric model turns to 2D model, and in three-dimensional model the computational domain is limited by two symmetry planes and includes two inlet 
ports by means of that a quarter part of the valve may be considered only.

The axisymmetric model FVM (Finite Volume Method) is shown in Fig. 3a. The model was built from 7968 two-dimensional elements and has 8690 nodes. The eight inlet holes were replaced by one equivalent inlet channel having the same flow surface as the sum of all surface inlet holes of the valve.

The three-dimensional model shown in Fig. $3 \mathrm{~b}$ represents a quarter part of the real valve and allows to model a real behaviour of inlet flow. Inlets reflect the actual shape and location. A three-dimensional model was assumed as reference model [7] for further analysis. This model is more complicated then axisymmetric one and has been described by 110454 spatial elements and 34437 nodes.

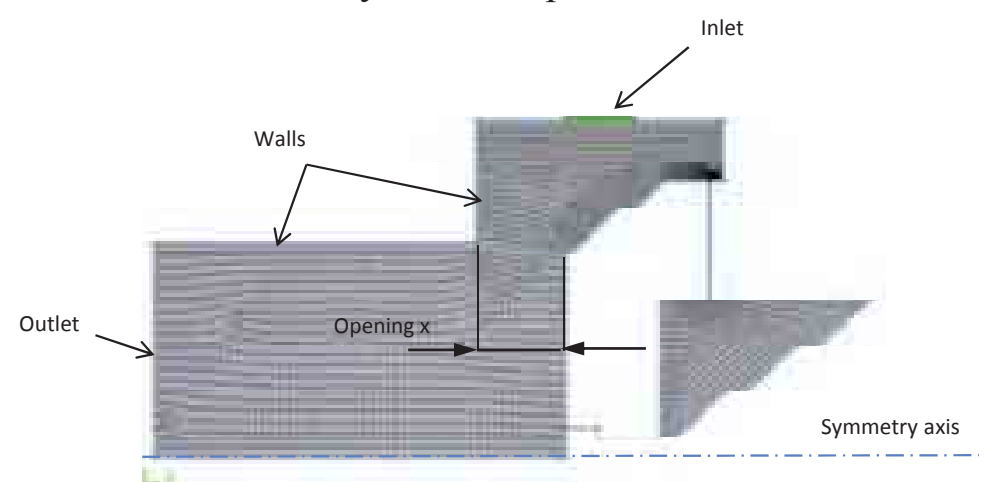

a)

b)

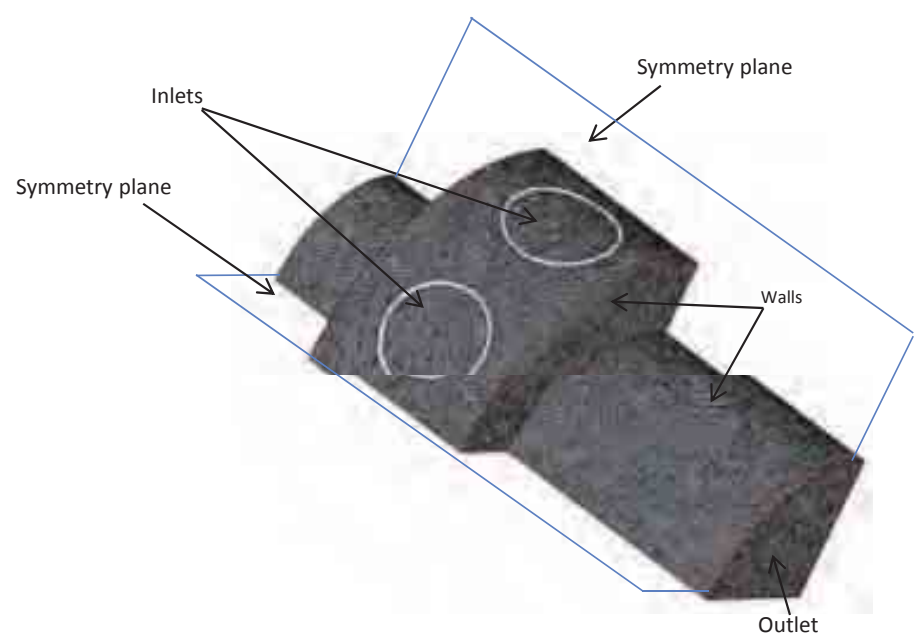

Fig. 3. The computational grid in the modelled geometry: a) axisymmetric model 2D, b) 3D three-dimensional model

The inlet velocity of the working medium is defined by the expression:

$$
\begin{aligned}
& V=\frac{Q}{S}, \\
& S=L \pi d,
\end{aligned}
$$

where:

$\mathrm{Q}$ - volumetric flow rate $\left[\mathrm{m}^{3} / \mathrm{s}\right]$,

$\mathrm{V}$ - inlet flow velocity $[\mathrm{m} / \mathrm{s}]$,

$\mathrm{S}$ - surface of inlet flow area $\left[\mathrm{m}^{2}\right]$.

As the working medium, hydraulic oil was assumed. The fluid density and the kinematics viscosity have been set to $\rho=870[\mathrm{~kg} / \mathrm{m} 3]$ and to $v=1.6 \cdot 10-5[\mathrm{~m} 2 / \mathrm{s}]$, respectively.

Simulation analysis was carried out using the commercial "Advanced CFD" package. For numerical solving the problem, the Euler domain based approach was selected where the fluid is 
moving towards to fixed grid.

The analysis was performed for a steady-state flow $[4,5]$, where the flow parameters such a velocity and pressure are time independently in metering sections.

\section{Numerical analysis and results}

Presented results allow to estimate differences between two considered models of control valve. The analysis was performed for seven values of flow rates Q: 5, 10, 15, 29, 25, 30, 40 [1/min] at the full valve opening (flow orifice $\mathrm{x}=2 \mathrm{~mm}$ ).

Figure 5 and 6 show the maps of pressure distribution and flow velocity for three-dimensional and axisymmetric model, in three cases of volumetric flow rate: 10 1/min, $201 / \mathrm{min}, 301 / \mathrm{min}$ volumetric.

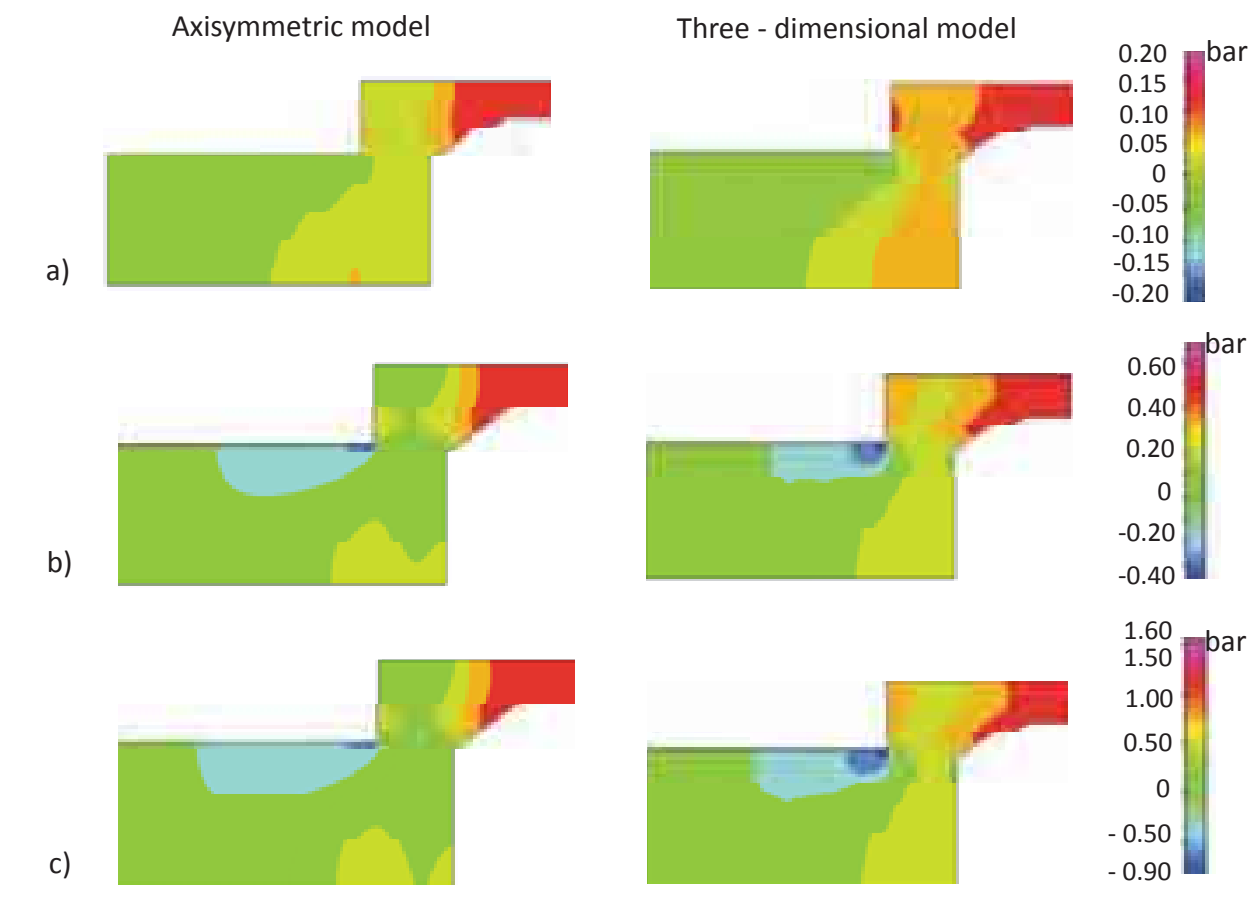

Fig. 4. Pressure distribution for axisymmetric and three-dimensional mode for different flow rate: a) $10 \mathrm{l} / \mathrm{min}$, b) $20 \mathrm{l} / \mathrm{min}, \mathrm{c}) 30 \mathrm{l} / \mathrm{min}$

Comparing the maps in Fig. 4 it can be stated that the pressure distribution inside the valve resulted both axisymmetric and three-dimensional model is very similar.

The area with negative values of pressure is observed for both cases in the wall of valve body close to orifice edge. Of course it does not meet the real situation, because results from the assumed boundary condition OUTLET $p=0$. For the great value of the outlet pressure, the pressure distribution in metering section will increase appropriately. However, such significant local pressure drop points out to danger of the appearance of cavitations, which is fast growing along with the growth of flow rate.

Figure 5 shows the velocity distribution maps of the fluid flow for the axisymmetric and threedimensional model. We observe the greatest speed streams in the central area of the streamlines, and the lowest ones close to the valve walls. Like to pressure distribution, in this case also we can state a good consistency between both models. The velocity distribution on the outlet is a bit more homogeneous for three-dimensional model.

We can see in Fig. 6 that for three-dimensional model the streamlines are found more regular see the areas in which fluid flow is formed as the steady-state flow. The both models show a local fluid vortexes appearing close to wall of poppet. For axisymmetric model, the additional vortex close to wall of outlet channel is found, but it results of model simplifications regarding to inlet channel, because a such phenomena is not observed for three dimensional model. 
a)

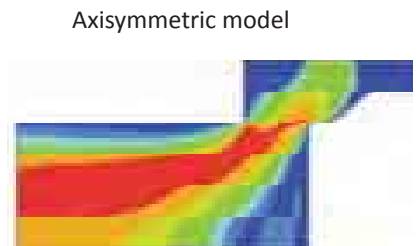

b)

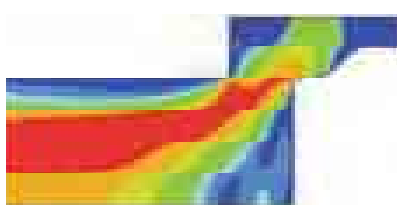

c)

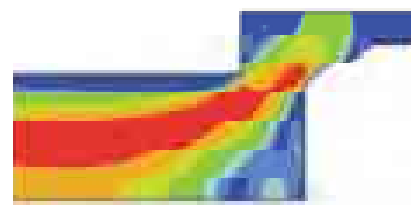

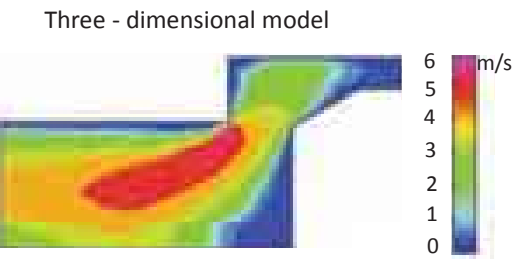
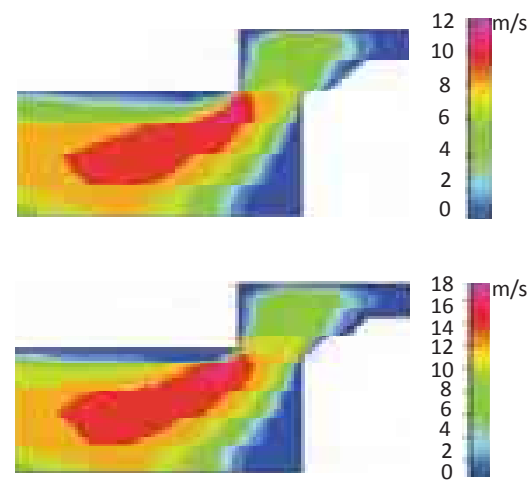

Fig. 5. Fluid velocity distribution in valve metering section for axisymmetric and three-dimensional model for different flow rates: a) $10 \mathrm{l} / \mathrm{min}$, b) $20 \mathrm{l} / \mathrm{min}, \mathrm{c}) 30 \mathrm{l} / \mathrm{min}$

For selected flow rate $\mathrm{Q}=20 \mathrm{l} / \mathrm{min}$ an example of streamlines for both models is shown in Fig. 6.

Axisymmetric model

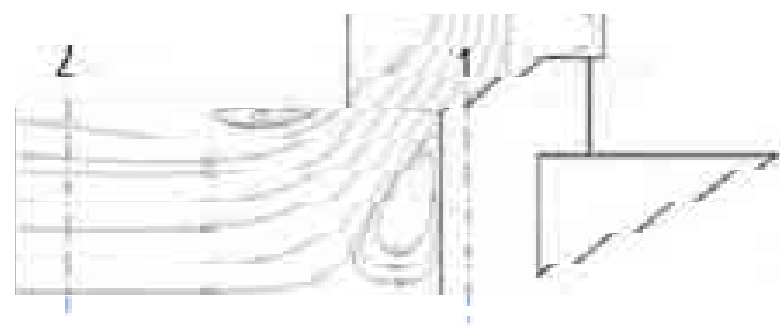

Three - dimensional model

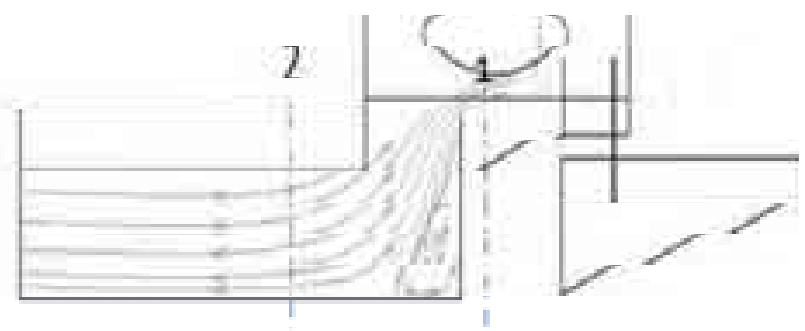

Fig. 6. The example of streamlines for volumetric flow rate $Q=20 \mathrm{l} / \mathrm{min}$

On the basis of pressure, distribution in inlet and outlet metering sections it is possible to calculate a corresponding mean pressure values in this sections and then the total pressure drop $\Delta \mathrm{p}_{\mathrm{d}}$ across the valve is expressed as:

$$
\Delta \mathrm{p}_{\mathrm{d}}=\mathrm{p}_{\text {in }}-\mathrm{p}_{\text {out }},
$$

where: $p_{\text {in }}, p_{\text {out }}-$ mean pressure in the inlet and outlet metering section respectively.

A relationship between pressure drop and flow rate defines a base flow characteristic of the valve $\mathrm{p}_{\mathrm{d}}=\mathrm{f}(\mathrm{Q})$.

The comparison of flow characteristics obtained from simulation results as well as experimental data is shown in Fig. 7. The both models give good results compared to the real flow in the valve. For the considered cases, the flow is turbulent and the flow curves are nonlinear. The largest pressure drop $\Delta \mathrm{P}_{\mathrm{d}}=1,42$ [bar] is observed for the largest volumetric flow rate $\mathrm{Q}=40[1 / \mathrm{min}]$.

For small values of volumetric rate flow, from 0 to $151 / \mathrm{min}$, practically there are no differences between 2D and 3D characteristics and they demonstrate the same variation towards to experimental curve, showing the pressure drop in the axisymmetric and three-dimensional model. In this case, the influence of the geometry of inlet metering section on the simulation results is negligible small. 


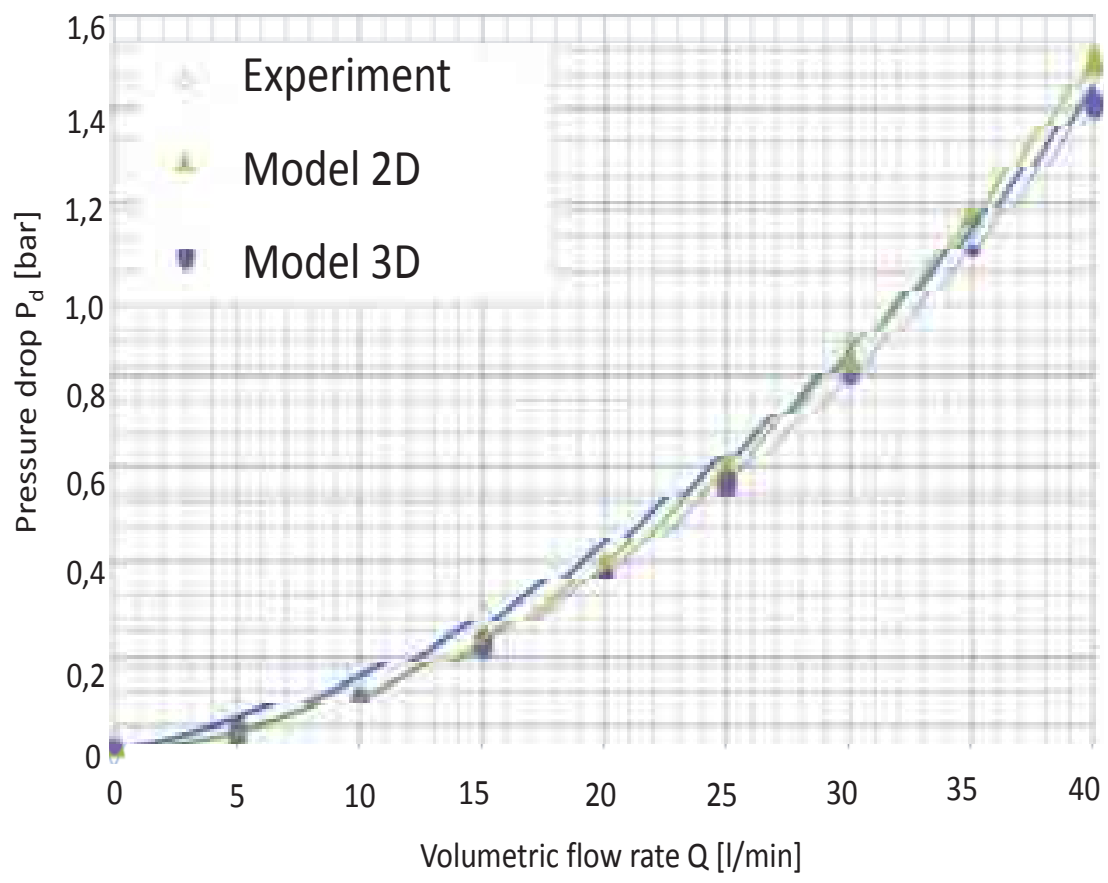

Fig. 7. The comparison of flow characteristics for the axisymmetric model, three-dimensional model and experiment

For large values of volumetric rate flow, over $151 / \mathrm{min}$, the gap between $2 \mathrm{D}$ and 3D characteristics increases because of the growth of a viscous forces between fluid layers and between fluid and valve walls. This phenomenon is more accurately described by 3D model. Maximum difference in pressure drop between 3D and axisymmetric models is observed for maximum flow rate but does not exceed $5 \%$ for flow rate $40 \mathrm{l} / \mathrm{min}$. It can be stated that for design practice the axisymmetric model is enough accurate.

\section{Summary and conclusions}

The paper presents a comparison the simulation results obtained from CDF analysis of on-off poppet control valve using two different numerical models - axisymmetric and three-dimensional.

The validation of numerical models was based on the experimental flow characteristics of the poppet control valve 2URES6 provided by the manufacturer [2].

The comparison of the pressure fields and flow velocity distribution shows small differences between the both models, which result from the modelling way of valve inlet channel. In the case of axisymmetric model, the simplification of the inlets geometry gives a good numerical efficacy with computation time $20 \mathrm{~min}$ but impacts on the fluid streamlines. In the case of three-dimensional model, the computations take 2208 min but the keeping count of real geometry of valve inlet holes cause that the streamlines (fluid element paths) inside the valve seem to be more realistic.

The comparison of the simulation and experimental flow characteristics confirmed a usefulness of the both models for subsequent analysis.

On the basis of numerical analysis, results it can be stated that:

- the both models give probable results compared to experimental data,

- numerical analysis based on axisymmetric 2D model is much more efficient then 3D model,

- simplifications introduced in the 2D model give a bit errors to be neglected,

- CFD analysis based on axisymmetric model has sufficient accuracy for engineering practice.

The simulation results have confirmed the good accuracy of the simplified CFD analysis based on 2D axisymmetric valve model being more efficient than full 3D approach and show this method as very useful to forecast in a design stage flow characteristics of poppet control valves. 


\section{Acknowledgements}

The authors wish to acknowledge the financial support of the European Commission under the Grant Agreement No. 232562 FP7-SME-2008-1, Project “HYDROCOAT”, FP7 Framework Programme.

\section{Reference}

[1] Bukowski, J., Mechanika płynów, PWN, Warszawa 1959.

[2] Karta katalogowa nabojowego zaworu rozdzielającego 2URES6. Ponar Wadowice S.A.

[3] Domagała, M., Metodyka modelowania zaworów maksymalnych, Praca Doktorska, Politechnika Krakowska, Instytut Informatyki Stosowanej, Kraków 2007.

[4] Yang, Y. S, Semini, C., Tsagarakis, N. G., Caldwell, Zhu, Y.,Water Hydraulics $-A$ novel Design of Spool Valves for Enhanced Dynamic Performance, Proceedings of the IEEE/ ASME, International Conference on Advanced Intelligent Mechatronics, Xian, China 2008.

[5] Yuan, Q.-H., Li, P.-Y., Using steady flow force for unstable valve design: modelling and experiments, Journal of Dynamic Systems, Measurement and Control, Vol. 127, No. 3, pp. 451-462, 2005.

[6] Guillion, M., Teoria i obliczanie układów hydraulicznych, WNT Warszawa 1961.

[7] Nowak, M. W., Metody identyfikacji układów ciagłych $z$ wykorzystywaniem funkcji modulujacych $i$ sklejanych $i$ ich zastosowanie $w$ regulatorze adaptacyjnym, Praca Doktorska, Akademia Górniczo-Hutnicza w Krakowie, 2007. 